of these figures, that mechanical filtration under these conditions can be firmly indorsed. ${ }^{1}$

The forcgoing paper and table of percentages was briefly discussed, first by Dr. P. H. Bryce, who thought the facts contained therein were most valuable and that he felt personally that the thanks of the Association were due to Dr. Swarts for submitting them, and by Dr. Rudolf Gering.

\section{THE SURGICAL TREATMENT OF AURAI POLYPI.}

BY P. B. WING, M.D.

TACOMA, WASHINGTON.

It is the purpose of this paper to describe in a brief and concise manner the treatment which $I$ have found most effectual for the permanent removal of those morbid growths known and described as aural polypi. Gruber defines aural polypus as a neoplasm originating in the auditory canal or in the deeper parts of the ear, which is pedunculated and consists principally of histologic elements of the soft connective tissue order. Granulations springing from a broader base are generally called polypoid proliferations. These growths have received a great variety of names by different writers, but I am of the opinion that no observer can say that this is a mucous polypus or a fibrous, but that he will find a combination of both mucous and fibroid tissue. I am also of the opinion that ail polypi are at the very commencement granulation tissue and that as they grow in size they change in structure, and that they have received their classification from the tissue that happened to be most prominent in each particular case; also that the epithelium covering a polyp has the character of the tissue from which it springs until it is changed by exposure to external influences. A word only as to the cause. I believe that whenever aural polypi and granulations are found, pus is always the cause, and when we come to treatment, we should never lose sight of this fact.

I shall confine myself to the treatment of those that are benign in character and shall make no mention of the tuberculous, syphilitic and malignant.

The first step in treatment is to thoroughly cleanse the ear. There is usually a profuse discharge of fetid pus, and it is for this discharge that patients usually seek relief, for unless the growth is large, the majority of patients are ignorant of its presence. After cleansing the ear as much as possible, the next step is to make out as accurately as we can the origin of the polyp. In small growths, this is usually easy, but in large polypi, that have filled and by long continued pressure caused absorption and dilatation of the canal, this is sometimes difficult and can only be made out approximately by passing a probe around it.

After we are satisfied as to the point of origin, the removal of the growth is the next point for consideration. How shall we best accomplish it? We are all armed with an instrument known as the snare, but I believe it falls far short of filling the purpose for which it was designed. The loop is formed and adjusted to the size of the tumor, and after applying cocain, or in some instances giving chloroform, the loop is passed over the tumor and as near the origin of the growth as possible. The loop is then pulled home. After pulling the loop tightly around the pedicle, Dr. Pomeroy says that he brings the growth

1 The complete report with tables and estimates of construction will be published in the Rhode Island State Board of Health Report for 1894 . away by two or three jerks. Dr. Sexton says that it is often difficult to cut through the pedicle and they often have to be brought away by avulsion. I feel like making a little stronger statement and say that it is always difficult to cut through the pedicle of a large fibroma, and that $I$ have always had to give the snare a few turns to twist off the growth. I see a reason why a suare can not be made that will work as well on aural as the Jarvis snare does on nasal polypi. After the polypus is removed, or as much of it as is possible with a snare, the hemorrhage, which is seldom severe, can be controlled by hot water or packing with absorbent cotton. I usually wait until the following day before I proceed to treat the stump. In the majority of cases, we are obliged to use some form of cautery, and in my opinion, no form of cautery is equal to chromic aoid.

A small bead of the crystals is fused on the end of a silver probe and applied under perfect illumination. After applying the acid, it is well to wait a few seconds watching the effect, and then touch the spot with a little moist cotton on a probe to take up any superfluous acid. I have used chromic acid in this manner for five years in a great number of cases and have found it perfectly safe. It gives almost no pain and its action is easily controlled. A surface treated with chromic acid suppurates little and heals rapidly. After cleansing and treating with chromic acid, I apply a little finely powdered iodoform and boric acid or hydrastis and boric acid and place a pledget of cotton in the ear. The chromic acid can be applied as often as the slough separates, usually every four or five days. The ear is kept clean during the intervals by using a solution of bichlorid of mercury, about 1 to 2,000, and after cleansing and drying, applying either of the above mentioned powders. This treatment is kept up until the pedicle is destroyed and when the growth is removed, the discharge in a large majority of the cases will cease. Small polypi are usually softer and bleed more easily than the large ones. The snare is perhaps more effectual in this class of cases.

However, there are very few cases treated by the snare that do not require subsequent cauterization. In small polypi, where the point of origin can be easily seen, the pedicle may be touched with chromic acid. The acid penetrates the growth, shuts off the blood supply, and in three or four days the polypus dries up, drops off and comes away as a small, black mass. This process is painless and bloodless, has the advantage of being easily done and is very convenient in treatment of children who are afraid of instruments.

Polypoid proliferations more often spring from the wall of the canal and, I think, are dependent upon some previous furuncle. These growths are to be removed by cauterization and I prefer the acids, used with the same precautions as in other places.

There is one condition resembling polypoid proliferation, which should not be confounded with it. I refer to a swollen and protruding mucous membrane of the middle ear, which may project through a perforated drum-head and be mistaken for granulation. Such cases are made worse by the application of any form of cautery. While speaking of cauterization I have not mentioned the galvano-cautery, for while I prefer it in all nose and throat work, I must say that I consider the acid superior in the treatment of the ear. It can be applied on a small wire, which does 
not obstruct vision. The electrode is larger, obstructs vision more and is not so easily handled. The acid in most cases is painless and can be applied without the patient knowing it.

I shall mention a few only of the drugs used in such cases. Iodoform and boric acid I have already mentioned as suitable dressings, but they have no influence over the growth of polypi. Tannic acid, alum, etc., are worse than useless. Argentic nitrate may be used to stop a discharge of pus, but I am sure that it stimulates the growth of polypi and of granulations. Hydrogen peroxid is useful only as a cleansing agent and is inferior to a solution of bichlorid of mercury. Notwithstanding all that has been said of alcohol, I believe that it has absolutely no influence over the growth of polypi or granulations, but it does lessen the discharge of pus. Injection of the growth with Monsel's solution is effective, if well done, but is painful and can be used on large poly pi only. Patients will ask us if the growth will return and we ask ourselves what shall be done to prevent a recurrence.

Now, I think we are all of the opinion that suppurative disease or caries is always the cause of polypi and of granulations. It is therefore necessary to stop the suppuration and remove all necrotic tissue before we can say that our patient is cured, for polypi and polypoid proliferations will not grow in dry soil like sage brush and cacti in our western deserts, but like toadstools, they require shade and moisture, and this moisture is pus.

Let us always put in practice that old, sound surgical principle that wherever there is pus, let it out if possible and establish free drainage. If the growth returns and if the perforation is small, we should not hesitate to make it larger and when necessary remove one or mole of the ossicles, thus establishing free drainage and giving us an opportunity to treat the diseased parts effectually ; always remembering, however, that it is not a trifling operation, but must be skilfully performed and subsequently carefully treated, for cases of meningitis following it are not unknown and $I$ think we are too apt to cover our failures and publish our successes.

\section{TUMOR OF THE BRAIN INVOLVING THE OCULAR NERVES.}

Specimen presented to the Medical Society of Washington, D. C. BY ClaARNCE R. DUfOUR, Phar. D., M.D. WASHINGTON, D. C.

Physician in Charge of Eye Department Eastern Dispensary; Ophthalmologist and Otologist to Sibley Memorial Hospital; Assistant in Eye pital, and Instructor in Oph thalmology and Otology, Georgetown Medical College.

Mrs. H., widow, age about 56 years was referred to me at the Woman's Clinic, in the summer of 1894, on account of her eyes. Upon examination I found a complete paralysis of the muscles supplied by the third, fourth and sixth nerves, an exophthalmus and optic atrophy, all on left side. I diagnosed the trouble as being in the brain and so told the daughter who accompanied her. I could obtain no specific history. She had been operated upon some months previous for empyema of left antrum, the opening being made in the cavity of second molar tooth; there was free drainage through the nostril when I saw her. I kept the antrum well washed out with sol. boric acid and gave her sat. sol. iodid of potas. sium, $10 \mathrm{gtt}$. three times daily, increasing one drop daily, until she was taking $30 \mathrm{gtt}$. three times daily. Any attempt to increase this amount produced such constitutional disturbance that it could not be done. She was kept on this treatment until complete symp. toms of iodism were manifested, when it was changed to $1.16 \mathrm{gr}$. bichlorid of mercury and $3 \mathrm{grs}$. potassium iodid three times daily, and continued for months with occasional intermiseion of a few days. No result whatever from the treatment. About the middle of the summer she began to complain of intense pain in her head, which at first yielded to anodype treatment but which soon became constant and nothing but morphia would allay. About this time there began to be symptoms of loss of sensation on side of face and anesthesia of cornea, indicating that the first division of the fifth nerve was being implicated. This condition continued, with no abatement of the symptoms; she had periods of hallucinations, and as her daughter was obliged to work away from home, and there being no one to leave the mother with, the latter was sent to the hospital. It was thought best to reopen the antrum so as to establish freer drainage; this was done, considerable pus escaping. It was then washed out with antiseptic solution two to three times daily. An examination of the urine was made with results as follows: Amber in color, cloudy ppt., acid reaction; small amount of aibumin, epithelial cells and pus were found. After operation on antrum the pain in head subsided for a few days but began again and continued with occasional periods of intermission. Sine was discharged from hospital in the early part of November. Her condition grad. ually became worse, her right eye began to show symptoms of incipient atrophy of the optic nerve and an external squint was manifested. She was able to go about until April 1895 when she had to go to bed. She grew worse, her mind wandered and word deafness became manifest. On May 3 she died. On the following morning the skull was opened and the brain removed; It was put into a 2 per cent. solution of formalin, and a few days later the examination revealed the following condition: Gumma of dura mater in the anterior part of left middle fossa; this involved by extension the left anterior temporo.sphenoidal lobe; the growth surrounded the internal carotid artery, exerting pressure on the cavernous sinus and involved the left optic nerve at the commissural origin; considerable edema of the left anterior sphenoidal lobe. A second and smaller gumma involved that part of the brain mass which constitutes the left olfactory convolution; the third and smallest lay in the angle at the right optic commissure. The bone around the first growth had lost its compact covering and had become porous; the dura was very adherent to the bone. The ocular conditions during life were cleared up, I think, satisfactorily by the findinge at the post-mortem. The cavernous sinus receives anteriorly the ophthalmic vein through the sphenoidal fissure, and on its inner walls is found the internal carotid artery and the sixth nerve; on its outer wall are the third, fourth and the first division of the fifth nerve.

The pressure exerted by the tumor upon the sinus explains the paralysis of the muscles and the anesthesia of cornea, etc., this pressure together with pressure upon the ophthalmic vein, accounts for the exophthalmus by preventing the return flow of blood through the angular and ophthalmic veins into the cavernous sinus. The pressure also being exerted 